\title{
Best Achievements in Clinical Thyroidology in 2020
}

\author{
Eun Kyung Lee ${ }^{1,2}$, Young Joo Park P.4 $^{3,4}$ \\ ${ }^{1}$ Center for Thyroid Cancer, National Cancer Center; ${ }^{2}$ Department of Cancer Biomedical Science, National Cancer Center \\ Graduate School of Cancer Science and Policy, Goyang; ${ }^{3}$ Department of Internal Medicine, Seoul National University College \\ of Medicine; ${ }^{4}$ Department of Molecular Medicine and Biopharmaceutical Sciences, Graduate School of Convergence Science \\ and Technology, Seoul National University, Seoul, Korea
}

This review highlights the most interesting research in thyroidology conducted in 2020. The publications of interest discussed below dealt with the following topics: thyroid dysfunction, risk of thyroid cancer, molecular diagnostics and new therapeutics for thyroid cancer, and thyroid disease in the coronavirus disease 2019 pandemic era.

Keywords: Thyroid diseases; Thyroid neoplasms; Thyroid hormone; Therapeutics; COVID-19; Molecular diagnostic techniques

\section{INTRODUCTION}

In 2020, clinical studies in thyroidology reported outstanding results. Specifically, intriguing questions about thyroid dysfunction and thyroid cancer were answered through well-designed, randomized clinical trials. This review summarizes the important research published in 2020 .

\section{METHODS}

Original, peer-reviewed research articles published between January 2020 and December 2020 were extracted through an independent literature review. A brief summary of these articles is presented along with their clinical utility or implications. The publications of interest discussed below dealt with the following topics: thyroid dysfunction, risk of thyroid cancer, molecular diagnostics and new therapeutics for thyroid cancer, and thyroid disease in the coronavirus disease 2019 (COVID-19) pandemic era.

\section{HEALTH IMPACTS OF SUBCLINICAL HYPOTHYROIDISM AND THE BENEFITS OF THYROID HORMONE SUPPLEMENTATION}

The impacts of subclinical hypothyroidism on cardiovascular morbidity and mortality and the benefits of levothyroxine (LT4) replacement remain inconclusive because only limited prospective cohort studies or randomized controlled trials have investigated LT4 replacement. In February 2020, Inoue et al. [1] sought to clarify to what extent subclinical hypothyroidism is associated with cardiovascular mortality in a representative sample of 9,020 adults in the United States enrolled in the National Health and Nutrition Examination Survey, and demonstrated that cardiovascular disease mediated $14.3 \%$ and $5.9 \%$ of the associations of subclinical hypothyroidism and high-normal thyroid-stimulating hormone (TSH) levels with all-cause mortality, respectively. This finding suggests that investigations are needed to examine the clinical benefits of medical interventions for people with elevated TSH levels. Following those results,

\section{Copyright $\odot 2021$ Korean Endocrine Society}

This is an Open Access article distributed under the terms of the Creative Commons Attribution Non-Commercial License (https://creativecommons.org/ licenses/by-nc/4.0/) which permits unrestricted non-commercial use, distribution, and reproduction in any medium, provided the original work is properly cited. 
two preliminary but important results were reported from double-blind, randomized controlled trials of LT4 replacement in subjects with subclinical hypothyroidism. Jabbar et al. [2] investigated the effects of LT4 in patients with subclinical hypothyroidism presenting with acute myocardial infarction, but failed to show any benefits for outcomes such as left ventricular function, adverse events, and quality of life after 52 weeks of LT4 treatment. Furthermore, de Montmollin et al. [3] reported that LT4 replacement failed to improve hypothyroid symptoms or tiredness scores at 1 year in subjects aged $\geq 65$ years with subclinical hypothyroidism $(4.6 \leq \mathrm{TSH} \leq 11.9 \mathrm{mU} / \mathrm{L})$ in a secondary analysis of the randomized, placebo-controlled Thyroid Hormone Replacement for Untreated Older Adults with Subclinical Hypothyroidism Trial (TRUST).

During pregnancy, maternal deficiency of thyroid hormone is associated with low birth weight [4]; however, the impact of subclinical hypothyroidism has remained unclear. A recent systematic review and individual-participant data meta-analysis of 48,145 mother-child pairs from 36 cohorts [5] provided evidence that maternal subclinical hypothyroidism during pregnancy $(n=1,275)$ was associated with a higher risk of small for gestational age (SGA; odds ratio [OR], 1.24; 95\% confidence interval [CI], 1.04 to 1.48 ) and lower birthweight, while isolated hypothyroxinemia $(n=929)$ was associated with a lower risk of SGA (OR, 0.7; 95\% CI, 0.55 to 0.91) and higher birthweight. There was an inverse, dose-response association of maternal TSH and free thyroxine (T4; even within the normal range) with birthweight, suggesting the rationale of thyroid function screening during the prenatal period for better postpartum outcomes.

\section{NEW THERAPEUTIC AGENTS FOR GRAVES' DISEASE}

Graves' disease is characterized by the presence of auto-antibodies that stimulate the TSH receptor, resulting in hyperthyroidism. The first-line treatment of thyrotoxicosis is thionamide, an anti-thyroid drug (ATD) [6]. A randomized trial investigated the efficacy of a blocking dose of ATD with LT4 replacement compared to ATD monotherapy dose titration. The primary outcome was the percentage of patients with normal TSH levels between 6 months and 3 years after treatment, and the secondary outcomes included adverse event frequency and remission/ relapse at 4 years. The study showed no evidence to suggest that blocking and replacement is associated with improved outcomes compared to monotherapy [6].

The conventional treatment modalities for Graves' disease have remained unchanged for the past 70 years [7]. Recently, novel therapeutic agents targeting the CD40-CD154 co-stimulatory pathway or the insulin-like growth factor I receptor (IGFIR) were developed [8]. Kahaly et al. [9] conducted an openlevel phase II proof-of-concept study of iscalimab, an antiCD40 monoclonal blocking antibody in 15 Graves' disease patients. Iscalimab induced euthyroid status in seven patients (47\%) with a 12 - to 20 -week treatment period, but four $(57 \%)$ of those patients relapsed after discontinuation. Twelve $(80 \%)$ patients showed at least one reversible adverse event with a mild to moderate degree of severity [9]. The efficacy of the IGF-IR inhibitor teprotumumab on Graves' orbitopathy (GO), which is a serious extrathyroidal manifestation of Graves' disease associated with activation of the IGF-IR pathway [10], also showed promising results [11]; a 24-week treatment course of IGF-IR led to a reduction in proptosis ( $\geq 2 \mathrm{~mm}$ ) in $78 \%$ of 41 patients versus $7 \%$ of 42 controls. It also resulted in better secondary outcomes with respect to the Clinical Activity Score (frequency of score $0-1: 59 \%$ vs. 21\%), diplopia (response in $68 \%$ vs. $29 \%$ ) and quality of life (GO-QOL overall score: 13.79 vs. 4.43) than placebo, with scarce adverse events [11].

\section{RISK OF THYROID CANCER}

The results from a population-based nested case-control study from Nordic population-based national cancer registry data (Denmark, Finland, Norway, and Sweden) were published in December 2020, demonstrating the impact of early-life risk exposures on the risk of thyroid cancer [12]. The thyroid cancer risk in offspring was analyzed in relation to maternal comorbidities and birth outcomes among 2,437 thyroid cancer cases (81.4\% with papillary thyroid cancer [PTC], 77.1\% women) matched with up to 10 non-cancer controls based on birth year, sex, and country, and county of birth. Postpartum outcomes (higher birth weight, congenital hypothyroidism, postpartum hemorrhage) and maternal comorbidities (diabetes before pregnancy, thyroid dysfunction, goiter, and benign thyroid neoplasms) were each associated with an increased risk of thyroid cancer in offspring. Of note, maternal thyroid comorbidity markedly increased the risk, with ORs of 67.36 (95\% CI, 39.89 to 113.76) for goiter, 22.50 (95\% CI, 6.93 to 73.06) for benign neoplasms, 18.12 (95\% CI, 10.52 to 31.20 ) for hypothyroidism, and 11.91 (95\% CI, 6.77 to 20.94) for hyperthyroidism. Fetal congenital hypothyroidism also showed high OR of 4.55 (95\% CI, 1.58 to 13.08). This study provides evidence to support an association between intrauterine exposures, particularly those 
related to maternal thyroid status during pregnancy, and later risk of thyroid cancer, although some genetic predisposition for thyroid disease could not be excluded.

Although, thyroid hormonal status is known to be associated with the risk of thyroid cancer, whether thyroid dysfunction plays a causal role in the development of cancer remains inconclusive. Tran et al. [13] provided additional evidence regarding that issue by demonstrating that both hyperthyroidism and hypothyroidism were associated with higher risks of thyroid cancer (pooled risk ratio, 4.49; 95\% CI, 2.84 to 7.12 for hyperthyroidism) (pooled risk ratio, 3.31; 95\% CI, 1.20 to 9.13 for hypothyroidism) as compared to euthyroidism in a meta-analysis of 13 million subjects from 15 studies. However, two recent results on the association of thyroid cancer with genetic variants related to thyroid function demonstrated contrary results. Zhou et al. [14] performed a genome-wide association study (GWAS) meta-analysis for 22.4 million genetic markers in up to 119,715 individuals from three famous studies - the Nord-Trøndelag Health Study (HUNT study), Michigan Genomics Initiative (MGI), and the ThyroidOmics consortium - and found 74 susceptible loci for TSH levels that explained $13.3 \%$ of its variance. Unexpectedly, phenome-wide association tests for the polygenic scores of the TSH variants showed an association between high TSH polygenic scores and low thyroid cancer risk, and two-sample Mendelian randomization analysis also suggested that the TSH variants associated with elevating levels could potentially reduce thyroid cancer risk in several independent populations. In a similarly designed GWAS meta-analysis in up to 72,167 European-descent individuals from the Breast Cancer Association Consortium and UK Biobank, Yuan et al. [15] showed that genetically predicted TSH levels (OR, 0.47; 95\% CI, 0.30 to 0.73 ) and hypothyroidism (OR, 0.7; 95\% CI, 0.51 to 0.98 ) were inversely associated with thyroid cancer. Although, these studies suggested that TSH and hypothyroidism may play a role in thyroid cancer, the causal relationship should be further elucidated in future studies.

\section{DIAGNOSIS OF THYROID NODULES AND CANCER}

The Bethesda classification defines the possibility of malignancy according to cytopathology findings derived from fine-needle aspiration for thyroid nodules [16]. Indeterminate cytology is a cumbersome category, with a difficult-to-define risk of cancer, and comprises approximately $20 \%$ of thyroid nodules. Recent molecular testing (gene panel tests) have been proposed to reduce the need for diagnostic lobectomy in patients with nodules with indeterminate cytology. Livhits et al. [17] investigated the effectiveness of molecular testing techniques, and in particular sought to determine whether an RNA test (Afirma genomic sequencing classifier) or a DNA-RNA test (ThyroSeq v3 multigene genomic classifier) offered superior performance in estimating the risk of malignancy of thyroid nodules with indeterminate cytology. In their randomized clinical trial of 346 patients with 372 indeterminate thyroid nodules, the prevalence of malignancy was $20 \%$. The RNA test and the DNA-RNA test showed no statistically significant difference in performance, including sensitivity (100\% vs. 97\%, respectively), specificity ( $80 \%$ vs. $85 \%$, respectively), and positive predictive value $(53 \%$ vs. $63 \%$ ) allowing half of patients with indeterminate nodules to avoid diagnostic surgery ( $51 \%$ vs. 49\%, respectively).

Advanced computing and imaging techniques are also being applied to the diagnosis of thyroid nodules. A systematic review and meta-analysis of 19 studies involving 4,781 nodules evaluated the efficacy and accuracy of machine learning-based diagnosis [18]. The diagnostic performance of deep learning-based diagnosis was comparable to that of radiologists (sensitivity, 0.87 [ $95 \% \mathrm{CI}, 0.78$ to 0.93 ] vs. 0.87 [95\% CI, 0.5 to 0.89 ]; specificity, 0.85 [ $95 \% \mathrm{CI}, 0.76$ to 0.91 ] vs. 0.87 [95\% CI, 0.81 to 0.91$]$; diagnostic OR, 40.12 [95\% CI, 15.58 to 103.33 ] vs. 44.88 [95\% CI, 30.71 to 65.57$]$ ). Radiomics-assisted diagnosis based on ultrasound imaging of thyroid lesions adequately predicted the risk of lymph node metastasis in PTC [19]. Imaging data were collected by three ultrasound instruments (GE, SuperSonic, and Kretztechnik) and analyzed by four models with quantitative indexes (statistical model, traditional radiomics model, nontransfer learning radiomics and transfer learning radiomics [TLR]). The TLR model achieved an area under the curve of 0.95 , indicating its accuracy for predicting in lymph node metastasis, and was validated in a different set from another hospital. By predicting the presence of neck lymph node metastasis, the TLR model can be applied to determine candidates for active surveillance of PTC [19].

\section{NOVEL THERAPEUTICS FOR THYROID CANCER}

Anaplastic thyroid carcinoma (ATC) remains one of the most aggressive and fatal solid tumors. Recently, a combination of dabrafenib and trametinib therapy demonstrated substantial survival improvement in patients harboring the $B R A F^{\mathrm{V} 600 \mathrm{E}}$ variation [20]. Furthermore, neoadjuvant $B R A F$-directed therapy 
showed the feasibility of complete resection and local disease control [21]. Based on these findings, the emerging use of targeted therapy, immunotherapy, surgery and radiation therapy might improve overall survival (OS) in patients with ATC [22]. In a single-institution (the University of Texas MD Anderson Cancer Center) cohort study of 479 patients with ATC over nearly 20 years, 1- and 2-year OS significantly increased from $35 \%$ and $18 \%$ in the 2000 to 2013 period to $47 \%$ and $25 \%$ in the 2014 to 2016 period, and $59 \%$ and $42 \%$ in the 2017 to 2019 period, respectively [23]. They found that a harmonious multidisciplinary approach could improve OS, with hazard ratios of 0.45 (95\% CI, 0.39 to 0.63 ) for targeted therapy, 0.58 (95\% CI, 0.36 to 0.94 ) for the addition of immunotherapy to targeted therapy, and 0.29 (95\% CI, 0.10 to 0.78 ) for surgery following neoadjuvant $B R A F$-directed therapy. The last group $(n=20)$ showed a 1-year survival of $94 \%$ with a median follow-up of 1.21 years. The researchers proposed a treatment algorithm for patients with ATC based on the staging and BRAF mutational status, and showed that preemptive genetic profiling and directed immunotherapy might lead to better treatment outcomes for patients with ATC, who are desperate due to the grave prognosis of the cancer.

Another disease entity that has lacked breakthrough therapies is radioactive iodine therapy-refractory differentiated thyroid cancer (DTC). Multikinase inhibitors targeting the growth factor signaling pathways (sorafenib and lenvatinib) were developed and approved as anti-cancer drugs for patients with advanced progressive DTC. Recently, novel target molecules including rearranged during transfection (RET), tropomyosin receptor kinase (TRK), and somatostatin receptors are emerging. The RET proto-oncogene encodes a transmembrane receptor tyrosine kinase that is constitutively activated in several types of cancers. Germline or somatic RET mutations were found in approximately $70 \%$ of medullar thyroid cancers, and RET fusions are found in fewer than $10 \%$ of DTC [24]. Selpercatinib, a selective RET inhibitor, proved its efficacy and safety in a phase 1-2 trial of 162 patients with RET-mutant medullary thyroid cancer (MTC) [24]. The response rate was 73\% (95\% CI, 62\% to $82 \%$ ) in 88 drug-naïve MTCs, and $69 \%$ (95\% CI, $55 \%$ to $81 \%$ ) and $79 \%$ (95\% CI, $54 \%$ to $94 \%$ ) in previously treated RET-mutant ( $n=55)$ and RET-fusion positive $(n=17)$ MTCs, respectively. Selpercatinib showed durable efficacy with mainly low-grade toxic effects. The most common adverse event (grade 3 or higher) was hypertension, which was observed in $21 \%$ of patients. This study suggests that effective molecular screening for RET mutations will be essential in selecting proper patients.
The fusion of the TRK gene with another gene occurs in DTC and ATC, and activates carcinogenesis and promotes progression. There are two Food and Drug Administration-approved drugs for DTC patients: larotrectinib [25] and entrectinib [26]. Cabanillas et al. [27] analyzed data from 28 patients with advanced metastasis harboring neurotrophin tyrosine receptor kinase $(N T R K)$ gene fusion pooled from two larotrectinib phase 1-2 clinical trials (NCT02122913 and NCT02576431). The objective response rate was $75 \%$ ( $95 \% \mathrm{CI}, 55 \%$ to $89 \%$ ) and the duration of response ranged from 1.9 to 41.0 months. Adverse events were mostly grade $1-2$. These findings suggested that larotrectinib was highly efficacious and its safety profile was favorable. Entrectinib, another NTRK inhibitor, showed a response in one out of five patients with thyroid cancer in an integrated analysis of three phase 1-2 trials (ALKA-372-001, STARTRK-1, and STARTRK-2) [26].

Somatostatin receptor type 2 (SSTR2) is highly expressed in tumor tissues [28]. Thakur et al. [29] suggested that SSTR2 may function as a promising target molecule in thyroid cancer. SSTR2 was expressed more intensively in thyroid cancer lesions than in normal tissues, and a radiolabeled analog of SSTR2 $\left({ }^{68} \mathrm{Ga}-\mathrm{DO}-\right.$ TA-TATE) showed higher uptake in thyroid cancer patients, particularly in Hürthle cell thyroid cancer resistant to radioactive iodine therapy. Treatment with ${ }^{177} \mathrm{Lu}$-DOTA-EB-TATE, with higher theragnostic efficacy, reduced tumor size and extended survival in a mouse model. This novel radiolabeled ana$\log$ of SSTR2 has the potential to be implicated in the treatment and diagnosis of resistant thyroid cancer.

\section{THYROID DISEASE IN THE COVID-19 ERA}

The outbreak of COVID-19, caused by severe acute respiratory syndrome coronavirus 2 (SARS-CoV-2), is causing overwhelming challenges for health systems [30]. Several retrospective reports have described the prevalence of thyroid-related diseases and investigated changes in treatment strategies as well as access to medical services. Lania et al. [31] analyzed thyroid function in 287 patients hospitalized with COVID-19 infection in a non-intensive care unit setting to determine whether this infection contributed to abnormalities in thyroid function. Seventythree patients (25.4\%) had thyroid dysfunction, including thyrotoxicosis in 58 patients and hypothyroidism in 15 patients. Low TSH levels were associated with high levels of the inflammatory cytokine interleukin-6, suggesting that COVID-19 may be associated with a high risk of thyrotoxicosis [31]. A similar study was also performed in China. Chen et al. [32] reported 
that low TSH levels were present in $56 \%$ of patients with COVID-19. After recovery, the thyroid hormone levels of COVID-19 patients and control groups were not significantly different. Muller et al. [33] evaluated the frequency of subacute thyroiditis in COVID-19 patients requiring high intensity of care units (HICUs) or low intensity of care units (LICUs) as compared to non-COVID patients admitted to HICUs in Italy. Their study demonstrated that 93 COVID-19 patients in HICUs had lower TSH levels and higher C-reactive protein levels than non-COVID-19 patients in HICUs or COVID-19 patients in LICUs initially. Although more research is needed, these studies suggest that COVID-19 is associated with systemic immune activation that may possibly cause thyroid inflammation and result in hyperthyroidism or thyroiditis.

\section{CONCLUSIONS}

There have been rapid advances in understanding thyroid diseases in the last decades. This has been the result of breakthroughs in computational and genetic technology and the fruit of tremendous demands for personalized medicine. Endocrinology and Metabolism looks forward to publishing excellent and promising results in this field in 2021.

\section{CONFLICTS OF INTEREST}

No potential conflict of interest relevant to this article was reported.

\section{ORCID}

Eun Kyung Lee https://orcid.org/0000-0003-0098-0873

Young Joo Park https://orcid.org/0000-0002-3671-6364

\section{REFERENCES}

1. Inoue K, Ritz B, Brent GA, Ebrahimi R, Rhee CM, Leung AM. Association of subclinical hypothyroidism and cardiovascular disease with mortality. JAMA Netw Open 2020;3: e1920745.

2. Jabbar A, Ingoe L, Junejo S, Carey P, Addison C, Thomas H, et al. Effect of levothyroxine on left ventricular ejection fraction in patients with subclinical hypothyroidism and acute myocardial infarction: a randomized clinical trial. JAMA 2020;324:249-58.

3. de Montmollin M, Feller M, Beglinger S, McConnachie A,
Aujesky D, Collet TH, et al. L-thyroxine therapy for older adults with subclinical hypothyroidism and hypothyroid symptoms: secondary analysis of a randomized trial. Ann Intern Med 2020;172:709-16.

4. Korevaar TIM, Medici M, Visser TJ, Peeters RP. Thyroid disease in pregnancy: new insights in diagnosis and clinical management. Nat Rev Endocrinol 2017;13:610-22.

5. Derakhshan A, Peeters RP, Taylor PN, Bliddal S, Carty DM, Meems M, et al. Association of maternal thyroid function with birthweight: a systematic review and individual-participant data meta-analysis. Lancet Diabetes Endocrinol 2020; 8:501-10.

6. Wood CL, Cole M, Donaldson M, Dunger DB, Wood R, Morrison N, et al. Randomised trial of block and replace vs dose titration thionamide in young people with thyrotoxicosis. Eur J Endocrinol 2020;183:637-45.

7. Lane LC, Cheetham TD, Perros P, Pearce SHS. New therapeutic horizons for Graves' hyperthyroidism. Endocr Rev 2020;41:873-84.

8. Carballido JM, Regairaz C, Rauld C, Raad L, Picard D, Kammuller M. The emerging jamboree of transformative therapies for autoimmune diseases. Front Immunol 2020;11: 472.

9. Kahaly GJ, Stan MN, Frommer L, Gergely P, Colin L, Amer A, et al. A novel anti-CD40 monoclonal antibody, iscalimab, for control of graves hyperthyroidism-a proof-of-concept trial. J Clin Endocrinol Metab 2020;105:dgz013.

10. Kahaly GJ. Management of graves thyroidal and extrathyroidal disease: an update. J Clin Endocrinol Metab 2020; 105:3704-20.

11. Douglas RS, Kahaly GJ, Patel A, Sile S, Thompson EHZ, Perdok R, et al. Teprotumumab for the treatment of active thyroid eye disease. N Engl J Med 2020;382:341-52.

12. Kitahara CM, Slettebo Daltveit D, Ekbom A, Engeland A, Gissler M, Glimelius I, et al. Maternal health, in-utero, and perinatal exposures and risk of thyroid cancer in offspring: a Nordic population-based nested case-control study. Lancet Diabetes Endocrinol 2021;9:94-105.

13. Tran TV, Kitahara CM, de Vathaire F, Boutron-Ruault MC, Journy N. Thyroid dysfunction and cancer incidence: a systematic review and meta-analysis. Endocr Relat Cancer 2020;27:245-59.

14. Zhou W, Brumpton B, Kabil O, Gudmundsson J, Thorleifsson G, Weinstock J, et al. GWAS of thyroid stimulating hormone highlights pleiotropic effects and inverse association with thyroid cancer. Nat Commun 2020;11:3981.

Copyright (C) 2021 Korean Endocrine Society 
15. Yuan S, Kar S, Vithayathil M, Carter P, Mason AM, Burgess $\mathrm{S}$, et al. Causal associations of thyroid function and dysfunction with overall, breast and thyroid cancer: a two-sample Mendelian randomization study. Int J Cancer 2020;147: 1895-903.

16. Bai Y, Kakudo K, Jung CK. Updates in the pathologic classification of thyroid neoplasms: a review of the world health organization classification. Endocrinol Metab (Seoul) 2020; 35:696-715.

17. Livhits MJ, Zhu CY, Kuo EJ, Nguyen DT, Kim J, Tseng $\mathrm{CH}$, et al. Effectiveness of molecular testing techniques for diagnosis of indeterminate thyroid nodules: a randomized clinical trial. JAMA Oncol 2021;7:70-7.

18. Xu L, Gao J, Wang Q, Yin J, Yu P, Bai B, et al. Computeraided diagnosis systems in diagnosing malignant thyroid nodules on ultrasonography: a systematic review and metaanalysis. Eur Thyroid J 2020;9:186-93.

19. Yu J, Deng Y, Liu T, Zhou J, Jia X, Xiao T, et al. Lymph node metastasis prediction of papillary thyroid carcinoma based on transfer learning radiomics. Nat Commun 2020;11: 4807.

20. Subbiah V, Kreitman RJ, Wainberg ZA, Cho JY, Schellens JHM, Soria JC, et al. Dabrafenib and trametinib treatment in patients with locally advanced or metastatic BRAF v600mutant anaplastic thyroid cancer. J Clin Oncol 2018;36:7-13.

21. Wang JR, Zafereo ME, Dadu R, Ferrarotto R, Busaidy NL, $\mathrm{Lu} \mathrm{C}$, et al. Complete surgical resection following neoadjuvant dabrafenib plus trametinib in BRAF(V600E)-mutated anaplastic thyroid carcinoma. Thyroid 2019;29:1036-43.

22. French JD. Immunotherapy for advanced thyroid cancers: rationale, current advances and future strategies. Nat Rev Endocrinol 2020;16:629-41.

23. Maniakas A, Dadu R, Busaidy NL, Wang JR, Ferrarotto R, $\mathrm{Lu} \mathrm{C}$, et al. Evaluation of overall survival in patients with anaplastic thyroid carcinoma, 2000-2019. JAMA Oncol 2020;
6:1397-404

24. Wirth LJ, Sherman E, Robinson B, Solomon B, Kang H, Lorch J, et al. Efficacy of selpercatinib in RET-altered thyroid cancers. N Engl J Med 2020;383:825-35.

25. Hong DS, DuBois SG, Kummar S, Farago AF, Albert CM, Rohrberg KS, et al. Larotrectinib in patients with TRK fusion-positive solid tumours: a pooled analysis of three phase 1/2 clinical trials. Lancet Oncol 2020;21:531-40.

26. Doebele RC, Drilon A, Paz-Ares L, Siena S, Shaw AT, Farago AF, et al. Entrectinib in patients with advanced or metastatic NTRK fusion-positive solid tumours: integrated analysis of three phase 1-2 trials. Lancet Oncol 2020;21:271-82.

27. Cabanillas ME, Drilon A, Farago AF, Brose MS, McDermott R, Sohal D, et al. 1916P Larotrectinib treatment of advanced TRK fusion thyroid cancer. Ann Oncol 2020;31 Supple 4:S1086.

28. Sun LC, Coy DH. Somatostatin receptor-targeted anti-cancer therapy. Curr Drug Deliv 2011;8:2-10.

29. Thakur S, Daley B, Millo C, Cochran C, Jacobson O, Lu H, et al. 177Lu-DOTA-EB-TATE, a radiolabeled analog of somatostatin receptor type 2 , for the imaging and treatment of thyroid cancer. Clin Cancer Res 2020 Dec 22 [Epub]. https://doi.org/10.1158/1078-0432.CCR-20-3453.

30. Yoo WS, Chung HK. The management of thyroid disease in COVID-19 pandemic. Int J Thyroidol 2020;13:65-71.

31. Lania A, Sandri MT, Cellini M, Mirani M, Lavezzi E, Mazziotti G. Thyrotoxicosis in patients with COVID-19: the THYRCOV study. Eur J Endocrinol 2020;183:381-7.

32. Chen M, Zhou W, Xu W. Thyroid function analysis in 50 patients with COVID-19: a retrospective study. Thyroid 2021;31:8-11.

33. Muller I, Cannavaro D, Dazzi D, Covelli D, Mantovani G, Muscatello A, et al. SARS-CoV-2-related atypical thyroiditis. Lancet Diabetes Endocrinol 2020;8:739-41. 